\title{
Long-term seafloor geomagnetic station in the northwest Pacific: A possible candidate for a seafloor geomagnetic observatory
}

\author{
H. Toh ${ }^{1}$, Y. Hamano ${ }^{2}$, and M. Ichiki ${ }^{3}$ \\ ${ }^{1}$ Department of Earth Sciences, University of Toyama, 3190 Gofuku, Toyama 930-8555, Japan \\ ${ }^{2}$ Department of Earth and Planetary Science, University of Tokyo, 7-3-1 Hongo, Bunkyo-ku, Tokyo 113-0033, Japan \\ ${ }^{3}$ Institute for Research on Earth Evolution, Japan Agency for Marine-Earth Science and Technology, \\ 2-15 Natsushima-Cho, Yokosuka, Kanagawa 237-0061, Japan
}

(Received May 2, 2005; Revised November 24, 2005; Accepted November 24, 2005; Online published June 2, 2006)

\begin{abstract}
For two years, geomagnetic variations have been measured at the seafloor in the northwest Pacific. The seafloor data consist of the geomagnetic vector field measured by a three-component fluxgate magnetometer and the absolute scalar total force measured by an Overhauser (1953) magnetometer with attitude measurements for both orientation and tilt. Using the attitude data, the geomagnetic data at a site in the northwest Pacific $\left(41^{\circ} 06^{\prime} 08^{\prime \prime} \mathrm{N}\right.$, $\left.159^{\circ} 57^{\prime} 47^{\prime \prime} \mathrm{E},-5580 \mathrm{~m}\right)$, hereafter referred to as NWP, were converted into the same reference frame as land and satellite measurements. Short-period variations of the converted vector data were examined by Hamano's (2002) global time domain analysis method, which showed compatibility of the seafloor geomagnetic observatory data with the existing land observatory network. The smooth and gradual change of the Earth's main field (i.e., the geomagnetic secular variation) was also found consistent with those predicted by the latest International Geomagnetic Reference Field (IGRF-10; IAGA, 2005) and by Ørsted Satellite (Olsen, 2002) for not only the scalar field but also the vector field. This means that observation of the geomagnetic vector secular variation is now feasible on the seafloor.
\end{abstract}

Key words: Long-term seafloor geomagnetic observation, secular variation, IGRF, satellite measurements, the Earth's main field, attitude data, scalar and vector geomagnetic fields.

\section{Introduction}

We are now at a new era in the history of measuring the geomagnetic field by scientific instruments. A series of geomagnetic satellites carrying fluxgate magnetometers and highly accurate positioning instruments have been recently launched into Low Earth Orbit (LEO) to form a miniconstellation which includes Ørsted (Neubert et al., 2001), CHAMP (Reigber et al., 2002) and SAC-C. The launch of these long-life geomagnetic satellites was an epoch-making event because it had been a long wait since the launch of the previous LEO vector geomagnetic satellite, MAGSAT (Langel et al., 1980). MAGSAT was launched more than a quarter century ago, and it unfortunately provided only a short-term dataset of less than one year.

Progress of the remote-sensing technique in observing the geomagnetic field has lead to intensive activity in modelling the geomagnetic field. For instance, the first result from Ørsted (Olsen, 2002) yielded an accurate set of internal Gauss coefficients up to 29 degrees in addition to their linear time dependence, viz., the geomagnetic secular variation, up to 13 degrees. A combination of the vector and scalar field values observed by multiple satellites was also fed back for construction of global models such as three recently released generations of IGRFs (IGRF-8 through

Copyright (c) The Society of Geomagnetism and Earth, Planetary and Space Sciences (SGEPSS); The Seismological Society of Japan; The Volcanological Society of Japan; The Geodetic Society of Japan; The Japanese Society for Planetary Sciences; TERRAPUB
IGRF-10; Mandea and Macmillan, 2000; Macmillan et al., 2003; IAGA, 2005). It is from IGRF-9 that the spatial resolution was improved to degree 13 .

The advent of long-life geomagnetic satellites, in turn, increases the importance of geomagnetic observation networks on the Earth's surface. Our knowledge of the ionospheric current system is now much improved by a combination of land-based and satellite geomagnetic data. It is essential for a better understanding of the geomagnetic field to collect both Earth-locked and Sun-locked view of the field at the same time. This implies that coordinated use of both satellite and ground-based geomagnetic data is the best option to yield reliable estimates of both the internal and external geomagnetic field at the Earth's surface. The biased distribution of the land geomagnetic observatories is now at a stage of improvement by extending the geomagnetic observatory network to the seafloor (Toh et al., 2004; Copley, 2004; Beranzoli et al., 2003; Langel et al., 1995; Barker and Barraclough, 1985).

The intent of this paper is to describe the abilities as well as the limitations of the newly developed seafloor geomagnetic station in the northwest Pacific, and to examine to what extent the seafloor geomagnetic data are reliable by comparison with presently available global models. In doing so, we will pay attention to the smooth temporal variations of the geomagnetic vector field. Because the seafloor geomagnetic data range from August, 2001 through July, 2003, the candidate models to be compared are restricted to 


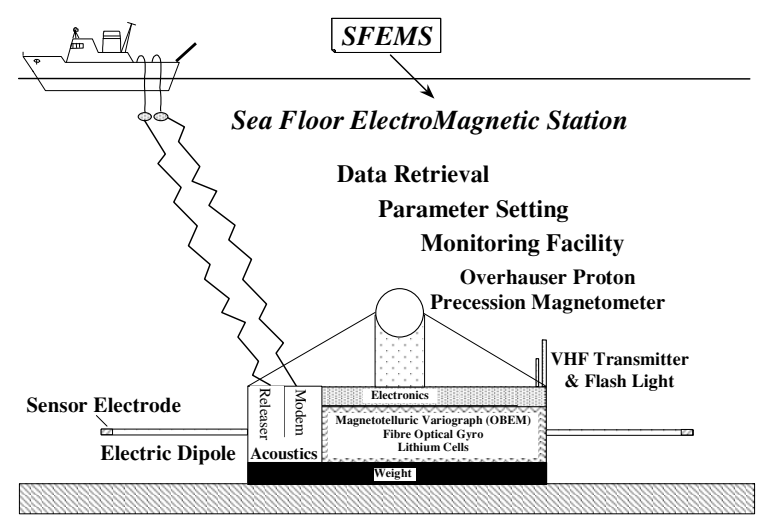

Fig. 1. Concept of the SeaFloor ElectroMagnetic Station (SFEMS). It is characterized by its absolute scalar magnetometer and acoustic modem that enables real-time communication between the sea surface and the seafloor. This instrument is also capable of measuring its attitude, i.e., precise variations of tilt and orientation with respect to the true north, and submersible to $6,000 \mathrm{~m}$.

the latest version of IGRF (IGRF-10) and that determined by Ørsted (Olsen, 2002) in order to enable comparison of the geomagnetic secular variation in addition to the field value itself.

\section{Instruments}

We developed a SeaFloor ElectroMagnetic Station (SFEMS) to carry out long-term geomagnetic observation in regions of poor data coverage. As shown in Fig. 1, SFEMS is a self-contained pop-up type system capable of unmanned operation for more than one year. Data retrieval via acoustic modem attached to SFEMS facilitates the uplink to the sea surface. However, the instrument needs to be manually retrieved to exchange the battery. The acoustic modem also provides functions of status monitoring of the seafloor instrument and/or resetting measurement parameters from the sea surface if necessary.

Detailed instrument packaging is described in Toh et al. (1998). However, we briefly summarize it here becuase a few updates have been made to SFEMS since 1998. SFEMS is able to conduct both scalar and vector measurements of the geomagnetic field. The absolute scalar measurements of the geomagnetic field were taken by an Overhauser (1953) proton precession magnetometer housed in a pressure-tight glass sphere located at height $1.85 \mathrm{~m}$ from the seafloor. The Overhauser magnetometer was proved to provide a minute-sampled scalar time series of the geomagnetic field within an absolute accuracy of $0.2 \mathrm{nT}$ (Toh and Hamano, 1997). The vector field measurement is taken by a three-component fluxgate magnetometer with a least count of $10 \mathrm{pT}$ for each axis. The fluxgate magnetometer is housed in another pressure-tight glass sphere mounted on a non-magnetic titanium frame at a level of $0.72 \mathrm{~m}$ above the seafloor. The other electronics, lithium primary cells and a fibre optical gyro (FOG) are contained in three separate glass spheres placed $0.61 \mathrm{~m}$ apart at the same level as the fluxgate magnetometer. The FOG axis is aligned with $\mathrm{X}$-axis of the fluxgate magnetometer. A major update of SFEMS since 1998 is the addition of the FOG and a triaxial transmitter coil system that enables vector geomagnetic measurements in the geographical coordinate system and in-situ calibration of the fluxgate magnetometer, respectively. Details of the new equipment will be described in the subsequent sections. It is also possible for SFEMS to provide magnetotelluric data by correlating the horizontal geomagnetic field and the two-component geoelectric field sensed by mutually orthogonal electric dipoles of $5 \mathrm{~m}$ long (see Fig. 1). However, the magnetotelluric result is beyond the scope of this paper, though delineation of the electrical conductivity structure beneath the very old seafloor around NWP is truly an interesting research topic.

The ultimate goal of SFEMS is to make it a seafloor geomagnetic observatory to be included in global geomagnetic field modelling. To achieve this, we need to establish a baseline control method for this unmanned system. Intermagnet (Kerridge, 2001), for instance, requires $1 \mathrm{nT} / \mathrm{y}$ accuracy of baseline changes of each geomagnetic component to fulfil the Intermagnet protocol in addition to semi-realtime data acquisition. Since SFEMS is a pop-up type instrument, the real-time distribution of acquired data is impossible in its present form, although it is partly enabled by the acoustic modem. We, therefore, tried to meet the baseline requirement by accurate monitoring of instruments' attitude at the seafloor.

The SFEMS's attitude measuring system consists of an FOG that intermittently measures the instrument's orientation at the seafloor, and a two-component tilt meter that continuously monitors the inclinations of the magnetic sensor from the horizontal plane. The tilt meter is packed with the fluxgate magnetometer to guarantee precise alignment of its axes with those of the magnetometer. We put the first priority on the tilt monitoring, although it is, of course, desirable to have continuous orientation monitoring as well. The reason is three-fold:

1) The geomagnetic potential can be determined by a combination of the vertical geomagnetic component and the horizontal force (or the total force measured by the Overhauser magnetometer).

2) The vertical geomagnetic component is of primary interest in the core surface flow determination using the detected geomagnetic secular variation (Roberts and Scott, 1965; Whaler, 1980; LeMouel, 1984; Voorhies and Backus, 1985).

3) FOG turned out to be very power-consuming and produced magnetic noise when activated.

The former two imply that determination of the geomagnetic potential and the core surface flow is made possible only after the instrument frame is truly rotated back to the horizontal plane. The continuity of the downward geomagnetic component can not be denied even in the presence of electric current sheets at the very surface of the perfectly conducting outer core. On the other hand, that of the horizontal components is only weakly guaranteed due to the current sheets, though vertical shears of the core surface flow can be estimated using the geomagnetic secular variations of the horizontal components (Jackson and Bloxham, 1991).

It was tedious work for us to construct an endurable attitude measuring system. It demanded, for instance, replace- 
Table 1. Temperature coefficients of each fluxgate vector magnetometer deployed at sea.

\begin{tabular}{cccc}
\hline & Northward [nT/deg] & Eastward [nT/deg] & Downward [nT/deg] \\
\hline Y2001-2002 & -2.2491 & +3.4632 & -1.4006 \\
Y2002-2003 & -2.4966 & -0.0653 & -4.5372 \\
\hline
\end{tabular}

Table 2. Scale factors and bias estimates of each fluxgate vector magnetometer deployed at sea.

\begin{tabular}{rrrrrrr}
\hline & \multicolumn{2}{c}{$x$-axis } & \multicolumn{2}{c}{$y$-axis } & \multicolumn{2}{c}{$z$-axis } \\
& \multicolumn{1}{c}{$a_{x}$} & \multicolumn{1}{c}{$b_{x}$} & $a_{y}$ & \multicolumn{1}{c}{$b_{y}$} & $a_{z}$ & $b_{z}$ \\
\hline Y2001-2002 & 0.9932 & $26.22 \mathrm{nT}$ & 1.0103 & $-0.28 \mathrm{nT}$ & 1.0076 & $31.06 \mathrm{nT}$ \\
Y2002-2003 & 1.0141 & $140.70 \mathrm{nT}$ & 0.9962 & $-19.10 \mathrm{nT}$ & 1.0062 & $164.20 \mathrm{nT}$ \\
\hline
\end{tabular}

ment of the tilt sensors a couple of times. Now, the attitude accuracy is precise to less than 5 arc seconds for the tilt measurement, while that of the orientation is still around 10 arc seconds or more. The former error gives the noise level of the tilt sensor used, while the latter is defined as the standard error of the mean computed from the real data, neglecting the temporal changes of the orientation. If a 30,000 nT magnetic field is applied to a magnetometer's axis, a tilt error of 7 arc seconds sums up to $1 \mathrm{nT}$ magnetic error. This means that a tilt precision better than 5 arc seconds is the necessary condition to clear the baseline change requirement of Intermagnet.

However, the orientation error of SFEMS is around 10 arc seconds, and it is not capable of detecting orientation changes with time. This is mostly due to logistical reasons that FOG is very power-consuming and produces magnetic noise when activated. Operations of FOG, therefore, were limited to once in three months for a typical one-year deployment of SFEMS. To improve the accuracy of orientation measurements better than 5 arc seconds, it is necessary to activate FOG once in three weeks, or adopt more accurate gyroscopes such as ring laser gyros. A detailed schedule how FOG was operated during the 2001-2003 observations will be described in Section 4.

\section{Sensor Calibration}

The sensor calibrations were conducted in May to June, 2001 and in June, 2002. Yatsugatake Magnetic Observatory (YAT), Earthquake Research Institute, University of Tokyo was chosen as the calibration site, and the whole system of the SFEMSs was calibrated there prior to each sea experiment by simulating the real configuration of the instruments at the seafloor. The calibrations were carried out in a nonmagnetic hut within YAT whose station corrections for each geomagnetic component were known. Table 1 lists temperature coefficients of the fluxgate vector magnetometers determined during each calibration. These coefficients were used in data processing of the acquired seafloor geomagnetic data.

Sensitivity and bias estimates of each axis of the vector magnetometers are summarized in Table 2. These estimates are derived by the following equation;

$$
\begin{aligned}
\text { YAT }_{i}= & a_{i} \times(\text { residual field })_{i} \\
& +(\text { primary canceling field })_{i}+b_{i}
\end{aligned}
$$

Table 3. Scale factor drifts determined by the in-situ calibration.

\begin{tabular}{llll}
\hline & $a_{x} / a_{x, 0}$ & $a_{y} / a_{y, 0}$ & $a_{z} / a_{z, 0}$ \\
\hline 2-DEC-2002 & 1.0013 & 1.0013 & 1.0009 \\
2-MAR-2003 & 0.9998 & 1.0003 & 1.0009 \\
\hline
\end{tabular}

where $a_{i}$ and $b_{i}$ are the scale factor and bias estimates of the $i$-th $(i=x, y$ or $z$ ) magnetometer axis, respectively. Raw readings of the vector magnetometers consist of the primary canceling field in units of hundred nanoteslas and the residual field within $\pm 328.76 \mathrm{nT}$, the former of which can be controled to a $0.01 \%$ accuracy. Temperature-corrected magnetometer readings and the absolute geomagnetic components in the hut $\left(\mathrm{YAT}_{i}\right)$ were substituted into Eq. (1) to yield the linear regression coefficients $a_{i}$ and $b_{i}$. Linearity of the vector magnetometers was found adequate for the simultaneous data of more than two weeks in each year.

However, we neglected instrumental drifts in Eq. (1). If there are significant temporal drifts in the magnetometer's 'residual field', they will appear as time-variant scale factors. To check for this, we added a triaxial transmitter coil to SFEMS in the 2002 deployment. The triaxial coil attached/aligned to FOG was designed to generate a magnetic dipole field of arbitrary direction with specified strength. The in-situ scale factor calibrations were then conducted for about $30 \mathrm{~min}$ after each FOG operation. The additional dipole field was rotated in XY- and XZ-plane of the measuring frame during the in-situ calibration. The calibration results are summarized in Table 3 in the form of scale factor ratios relative to the first in-situ calibration result. It is evident that the scale factors are stable for half a year and vary at most $0.13 \%$.

\section{Sea Experiments}

The building of SFEMS commenced in 1996. It was first applied to real observation in 1999 after three test deployments at sea (Toh and Hamano, 1997; Toh et al., 1998). However, the first significant geomagnetic data from SFEMS was not delivered until July, 2002 (Toh et al., 2004) due to a malfunction of SFEMS in the very first deployment for one year starting from August, 1999. We, therefore, were obliged to reinstall SFEMS in the northwest Pacific in July, 2001. Since then, we have succeeded in recovery of SFEMS twice, which has increased the total duration of 


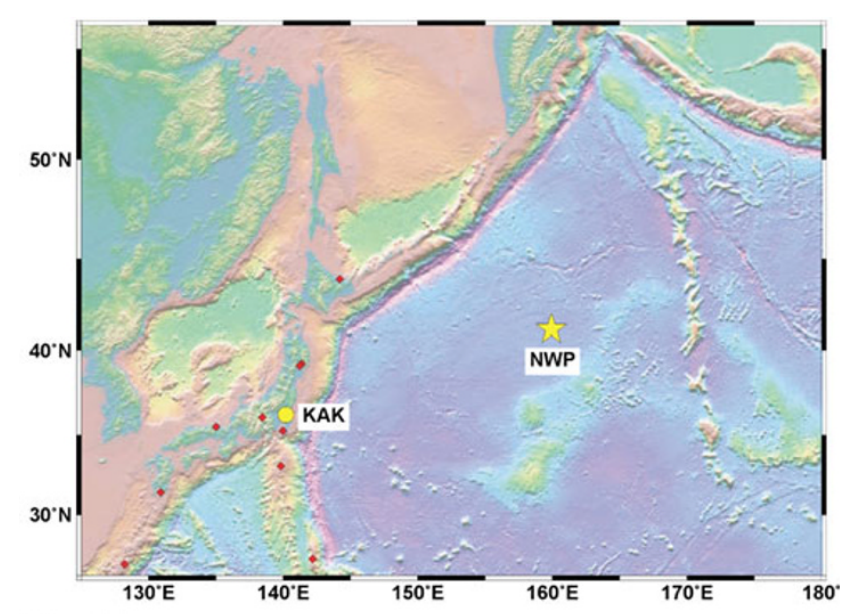

(1)

Fig. 2. Site map of the Northwest Pacific. The seafloor site, NWP, is located on the seafloor as old as $124 \mathrm{Ma}$ (Nakanishi and Winterer, 1998) and is about $1,800 \mathrm{~km}$ away from KAK.

obtained geomagnetic time series to 713 days for the vector measurement and 358 days for the scalar measurement by the same sampling rate of one minute.

NWP in Fig. 2 was chosen as the installation site for the long-term geomagnetic observation at the seafloor. One of the main reasons for site selection is that NWP is within the non-dipole low region known to exist in the Pacific. NWP is located about $1,800 \mathrm{~km}$ east of the closest geomagnetic observatory, Kakioka Magnetic Observatory (KAK; $36.23 \mathrm{~N}, 140.19 \mathrm{E})$; about halfway between the Midway Island $(28.21 \mathrm{~N}, 177.38 \mathrm{~W})$ and the Japanese Islands. NWP is situated on the seafloor as old as $124 \mathrm{Ma}$ (Nakanishi and Winterer, 1998).

The strong magnetic lineations, by which the age of the seafloor around NWP was determined, were further confirmed by an on-site surface tow of a proton precession magnetometer. The magnetic survey over NWP was conducted in the summer of 2003. The result of the surface tow is summarized in Fig. 3. Actual installation sites of the SFEMS slightly differ for each deployment as shown in the figure, although exact locations at the seafloor were determined within an accuracy of a few tens of meters by a combination of GPS navigation and acoustic ranging conducted after deployment. Figure 3 also shows that all installation sites evidently fall on a strong positive magnetic anomaly called the 'Japanese Lineation Set' (Nakanishi and Winterer, 1998), whose intensity at the sea surface is as large as $230 \mathrm{nT}$. This requires a considerably large 'station correction' for NWP.

The sea experiments around NWP consisted of three deployments and two recoveries of SFEMS in every summer since 2001. The time series obtained so far, therefore, is divided into a 338-day segment from 2001 through 2002 and a 380-day segment from 2002 through 2003 with a 5-day overlap. Prior to the deployments, schedules of FOG operations were preset into each SFEMS together with other measurement parameters such as the sampling rate, start time at the seafloor and so on. In the 2001-2002 deployment, FOG was scheduled to be activated three times, i.e., at

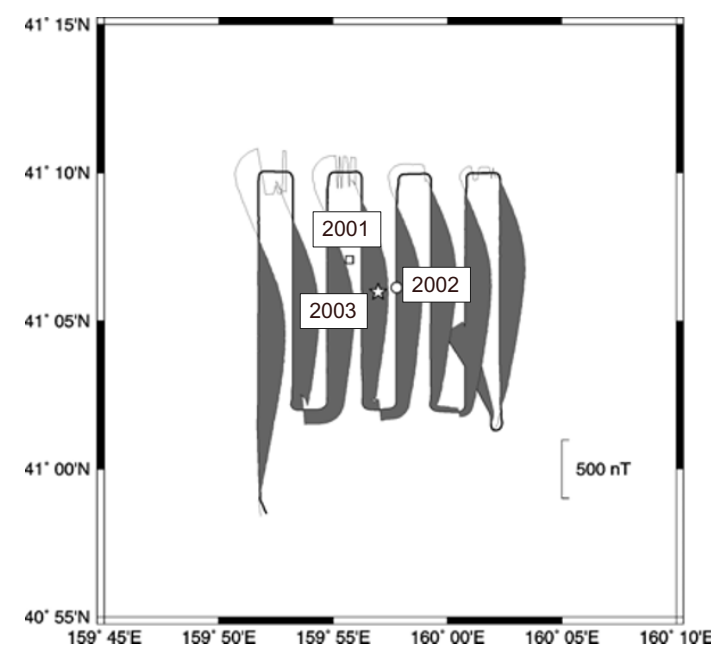

Fig. 3. Magnetic anomalies around NWP. It is evident that all the SFEMS are located in the strong magnetic anomaly called 'Japanese Lineation Set' (Nakanishi and Winterer, 1998). The symbols are the locations of SFEMS in each year.

00:00 UTC on 1-OCT-2001, 1-JAN-2002 and 1-APR-2002, respectively, while SFEMS itself started at 00:00 UTC on 1-AUG-2001. FOG was operated for 28 hours at each time, producing one orientation reading per minute. As for the 2002-2003 deployment, it was scheduled to operate on 1-SEP-2002, 1-DEC-2002 and 1-MAR-2003 with the same sampling rate and duration as in the 2001 deployment. SFEMS started recording the seafloor geomagnetic field at 00:00 UTC on 1-AUG-2001 and 15:00 UTC on 29-JUN2002 in the first and second deployments, repectively. After recovery, we measured SFEMS's clock gain/delay against ship's GPS clock in order to conduct clock adjustments in the subsequent data processing.

\section{Data}

The two-year long geomagnetic time series was further corrected for the clock gain/delay, temporal variations of temperature and tilts after removal of spikes occasionally seen in the raw data, and finally rotated back to the geographical coordinate using the estimate of the instrument's orientation at the seafloor. Correction for temperature variation was made using the temperature coefficients in Table 1. The SFEMSs are capable of measuring temperature variations in the fluxgate's glass sphere with a $0.01^{\circ} \mathrm{C}$ resolution. Since observed temperature variations are as small as $\pm 0.03^{\circ} \mathrm{C}$ within a year, the actual temperature correction summed up to at most $0.3 \mathrm{nT}$.

As for the tilt correction, we used the following formula to recover the horizontality of the observed vector geomagnetic data;

$$
\begin{aligned}
\left(\begin{array}{l}
X^{\prime} \\
Y^{\prime} \\
Z
\end{array}\right)= & \left(\begin{array}{ccc}
\cos \alpha & 0 & -\sin \alpha \\
0 & 1 & 0 \\
\sin \alpha & 0 & \cos \alpha
\end{array}\right) \\
& \times\left(\begin{array}{ccc}
1 & 0 & 0 \\
0 & \sqrt{1-\delta^{2}} & -\delta \\
0 & \delta & \sqrt{1-\delta^{2}}
\end{array}\right)\left(\begin{array}{l}
X_{o b s} \\
Y_{o b s} \\
Z_{o b s}
\end{array}\right)
\end{aligned}
$$

with

$$
\delta=\frac{\sin \beta}{\cos \alpha}
$$



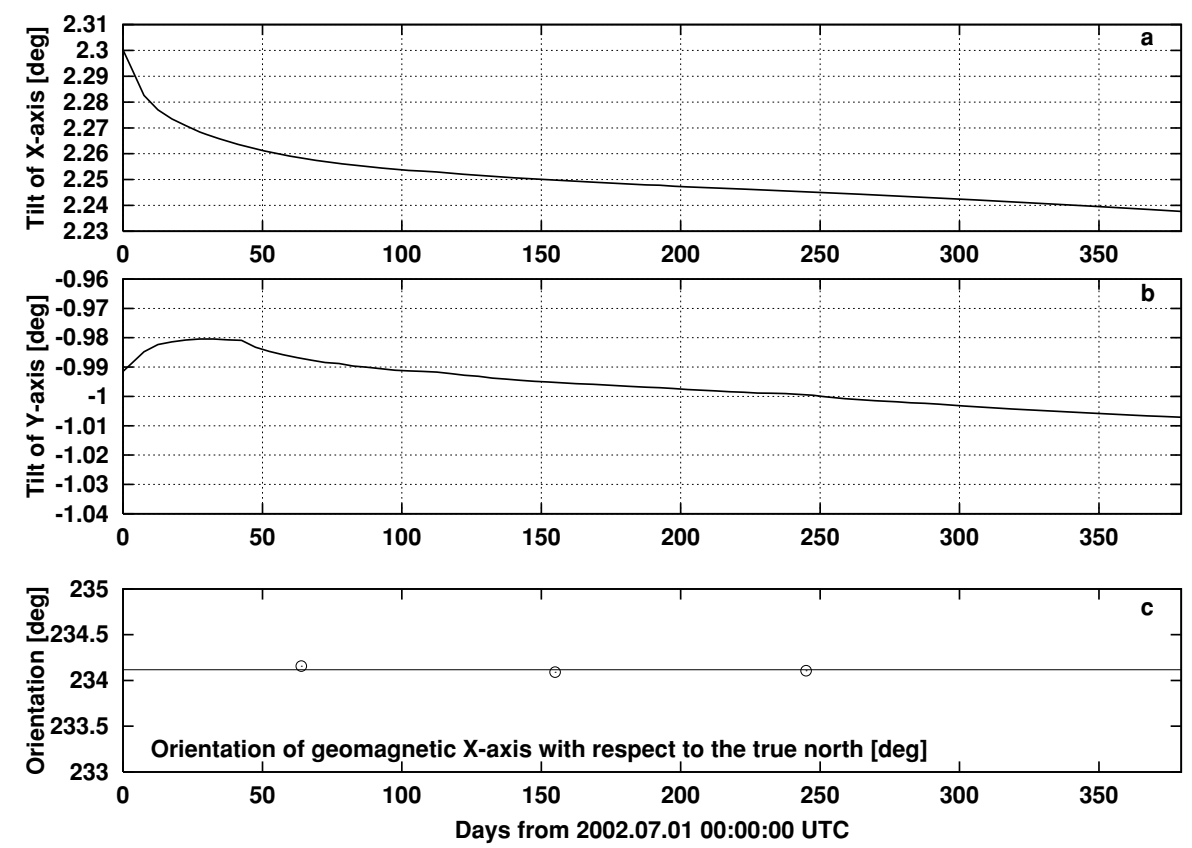

Fig. 4. The observed tilt changes for 380 days (Panels a and b), and the SFEMS's orientation at the seafloor (Panel c). The tilt components show down dip angles of each measuring axis from the horizontal plane. The horizontal line in Panel c indicates the overall average of N234. ${ }^{\circ} \mathrm{E}$. The 4 th FOG operation was originally planned at the time of recovery by sending commands from the ship via acoustic modem, which was not allowed due to rough sea state.

Table 4. Predicted and observed geomagnetic field at NWP on 23-SEP-2002*.

\begin{tabular}{ccccc}
\hline & Total Force [nT] & Northward [nT] & Eastward [nT] & Downward [nT] \\
\hline Satellite $^{\dagger}$ & $44,408.2$ & $26,618.2$ & $-1,352.2$ & $35,520.9$ \\
Seafloor $^{\dagger}$ & $45,254.8$ & $26,914.7$ & -614.0 & $36,138.1$ \\
\hline
\end{tabular}

* The geomagnetically quietest day during the seafloor observation. ${ }^{\dagger}$ Expanded by spherical harmonics up to degree 29. ${ }^{\ddagger}$ Daily means on $23-$ SEP-2002.

where vectors $\left(X^{\prime}, Y^{\prime}, Z\right)$ and $\left(X_{o b s}, Y_{o b s}, Z_{o b s}\right)$ are those in the horizontal frame and the instrument frame, respectively. $\alpha$ and $\beta$ are down dip angles measured by the tilt meter. Since this rotation preserves the orientation of the instrument's X-axis which was set aligned to that of FOG at the time of deployment, we had only to complete a final rotation of the $\left(X^{\prime}, Y^{\prime}, Z\right)$ vector in the horizontal plane using the FOG data in order to yield a $(X, Y, Z)$ vector in the geographical coordinate. As for the geoelectric data in which the vertical component was missing, we made an additional assumption that the tilt-corrected vertical geoelectric component was nil when the geoelectric vector was rotated back into the horizontal frame.

It was found that the magnetometer's $x$-axis at the seafloor pointed toward $\mathrm{N} 33.6^{\circ} \mathrm{E}$ for the 2001-2002 dataset and $\mathrm{N} 234.1^{\circ} \mathrm{E}$ for the $2002-2003$ dataset. It is noteworthy that the temporal variations of tilts shown in Figs. 4(a) and 4(b) were indispensable in order to recover horizontality of the instrument's frame as land observatories and satellite measurements. It was not until the smooth but everlasting changes of tilt were accurately monitored for over one year at the seafloor that the baseline changes of each component of the vector geomagnetic field were revealed with enough precision. Since the noise level of the tilt variation is smaller than 5 arc seconds and the maximum component of the vector geomagnetic field is that of $Z_{o b s}$ which is as large as $37,600 \mathrm{nT}$, the tilt correction error is at most 0.91 nT. This is small enough to detect the geomagnetic secular variation in the vertical component, which will be discussed in the next section.

On the contrary, temporal variation of orientation between each measurement is not resolved due to the too few number of measurements per deployment (Fig. 4(c)). Even if the temporal variation is neglected, the standard error of mean orientation is as large as 10.2 arc seconds, which implies at least $1.4 \mathrm{nT}$ ambiguity of horizontal components. It follows that detection of the secular variation in the horizontal components is not possible, while determination of the vertical secular variation and the geomagnetic potential itself are possible using the total force and the vertical component. Noise level of vector geomagnetic measurements during the FOG operations was found being enhanced to $1 \mathrm{nT}$ due to closer location of the fluxgate magnetometer to FOG, while that of scalar measurements was still within 0.2 nT, the absolute accuracy of the Overhauser magnetometer placed more than $1 \mathrm{~m}$ above FOG.

Table 4 compares four components of the geomagnetic main field actually observed at the seafloor with those predicted by the Ørsted Satellite (Olsen, 2002). As for the seafloor main field, a daily mean of the seafloor geomag- 

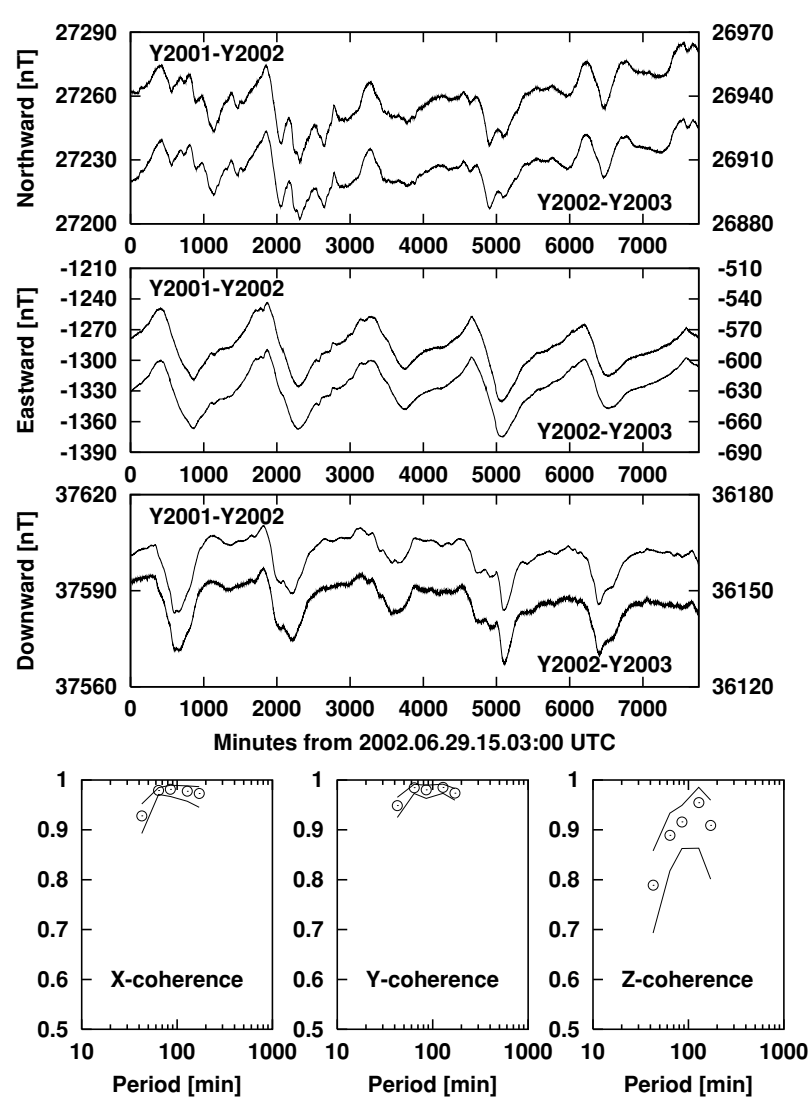

Fig. 5. Overlapped geomagnetic three components and their mutual coherences with $95 \%$ confidence intervals. Coherences of the horizontal components are larger than 0.9 for all periods, while that of the vertical component is decreasing toward short periods due to the higher noise level of the component during the 2002-2003 observation.

netic field was computed on 23-SEP-2002, one of the geomagnetically quietest days during the seafloor observation. The satellite estimates were calculated using the Gauss coefficients given up to 29 degrees and their first-order time derivatives up to 13 degrees. Downward continuation to a depth of $5580 \mathrm{~m}$ was also made for the satellite estimates. The difference between the averaged geomagnetic total force at the seafloor and the respective satellite estimate is less than $800 \mathrm{nT}$, which mainly stems from the smaller eastward component and larger downward component at the seafloor. The smaller eastward component may arise from the misalignment of the horizontal axes of the SFEMS's vector magnetometer with the true geographical north, since the eastward component is more sensitive to the misalignment than the northward component. The difference in the downward components is in agreement with the fact that the seafloor sites are located in the middle of the strong positive magnetic anomaly as described in the previous section. This means that 'station correction' at NWP may be at most $600 \mathrm{nT}$ and it mostly applies to correction for the downward component. It can not be denied that the satellite prediction used underestimates the lithospheric contribution at NWP, since only Gauss coefficients up to 29 degrees are incorporated. A sum of the magnetic signals of crustal origin with wavelengths shorter than $\sim 1400 \mathrm{~km}$ may be able to account for the significant part of the observed difference.

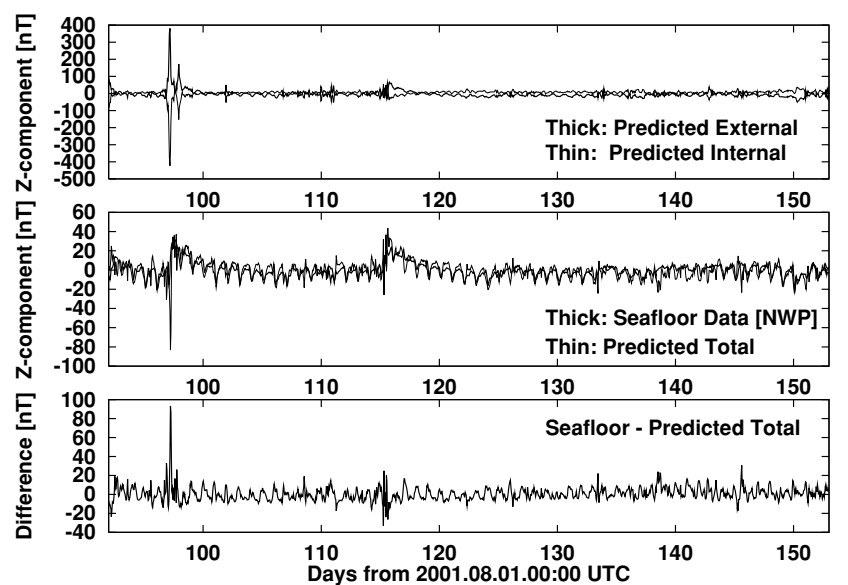

Fig. 6. Comparison of the downward geomagnetic variations at the seafloor with predictions by the global time domain analysis (Hamano, 2002). (Top) The predicted external and internal Z-component which are nearly anti-parallel. (Middle) The seafloor Z-component and the predicted total Z. (Bottom) Difference between the observation and the prediction.

As described before, installation of SFEMS was made by a freefall from the ship, which implies that the seafloor sites are different at each installation. However, by collecting simultaneous time series at both sites, the seafloor geomagnetic field differences from site to site can be acquired. Figure 5 shows a 5-day overlap of the seafloor geomagnetic variations for each component observed from the end of June through the beginning of July, 2002. Their mutual coherence estimates are also included in Fig. 5 with $95 \%$ confidence intervals, which were computed using a robust spectral analysis code (Chave et al., 1987). The temporal variations are coherent at the two different seafloor sites, while the baselines of each component significantly differ site by site. This is because the installation site of SFEMS in 2002 was shifted from the previous site as far as $3.7 \mathrm{~km}$ toward southeast (see Fig. 3 as well) due to a newly installed trans-ocean submarine cable from China to US. The differences of each baseline with respect to the latter installation were $352.61 \mathrm{nT}$ for the northward component, $-659.80 \mathrm{nT}$ for the eastward component and 1,454.55 nT for the downward component, respectively.

Compatibility of the temporal variations of the geomagnetic vector field observed at NWP was also examined using Hamano's (2002) global time domain analysis method. The method can provide stable estimates of internal and external Gauss coefficients of the temporal field with higher time resolution than usual spherical harmonic analyses. To achieve this, a stochastic inversion technique using singular-value decomposition was applied in order to yield temporal Gauss coefficients up to degree 6 from a dataset of hourly means of the northward and downward geomagnetic components at 45 worldwide observatories on land. Two successive geomagnetically active months (November to December, 2001) were chosen for the analysis. Eastward components were not included in the analysis, since inclusion of the eastward component is likely to make the whole inversion process unstable. The result of the global time domain analysis is shown in Fig. 6. The temporal geo- 

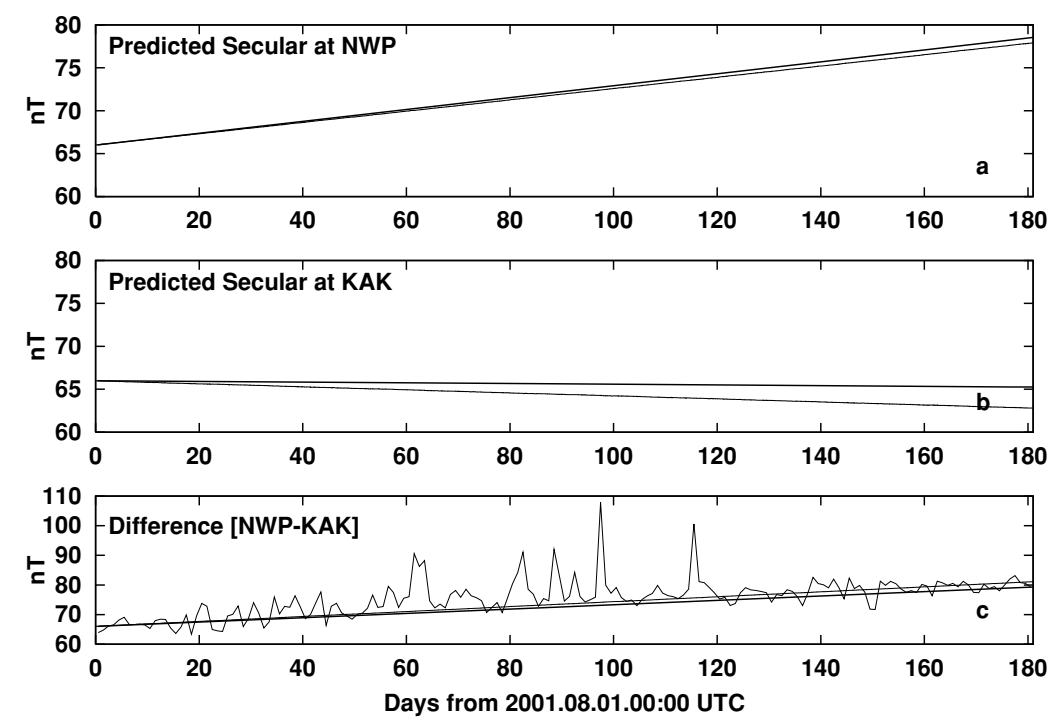

Fig. 7. Secular variation of the geomagnetic total force at NWP over a six month period. (a) Prediction by two global models. Since the two models give similar estimates at NWP, each prediction is undistinguishable. (b) At KAK. Prediction by IGRF-10 (thin line) and Ørsted (thick line). (c) Two global estimates are compared with the difference (the rugged thin line) of the observed scalar secular variations between NWP and KAK.

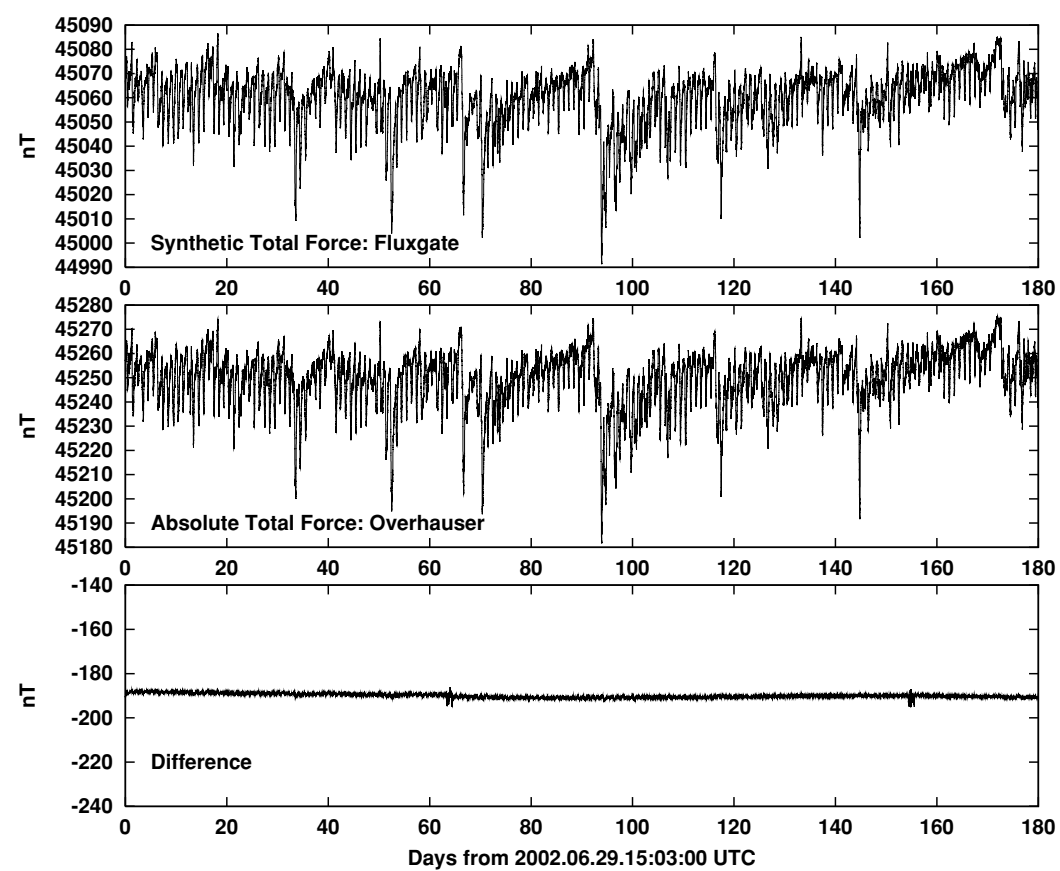

Fig. 8. (Top) Synthetic total force variations computed from the vector field measurement by the fluxgate magnetometer of SFEMS. (Middle) The absolute geomagnetic total force measured by the Overhauser magnetometer. (Bottom) Difference between the synthetic and absolute total force. Two disturbances are seen at the time of the FOG operations.

magnetic field of the downward component was computed and directly compared with that at the seafloor in the figure. Downward continuation to the seafloor was conducted for the predicted values again. It is readily seen that storm time variations with the form of Dst for two major storms in November and a smaller one at the end of December are reproduced very well at the seafloor using the data from the existing geomagnetic observatory network on land.

\section{Geomagnetic Secular Variation at the Seafloor}

It has been confirmed that the short-period temporal variations observed at NWP are compatible with those pre- dicted by the existing geomagnetic observatory network. If so, do long-period temporal variations of the scalar and vector fields also coincide with predictions by global models?

A gradual increase was observed in the absolute geomagnetic total force at NWP. Two global models, viz., IGRF-10 and the satellite estimate (Ørsted), predict almost the same increase at NWP as shown in Fig. 7(a). On the other hand, both models predict the similar decrease at KAK (Fig. 7(b)). As a result, both models show sufficient fits to the observed secular difference between NWP and KAK (Fig. 7(c)).

It is much more challenging to identify the secular variation in the vector field by an unmanned system such as 

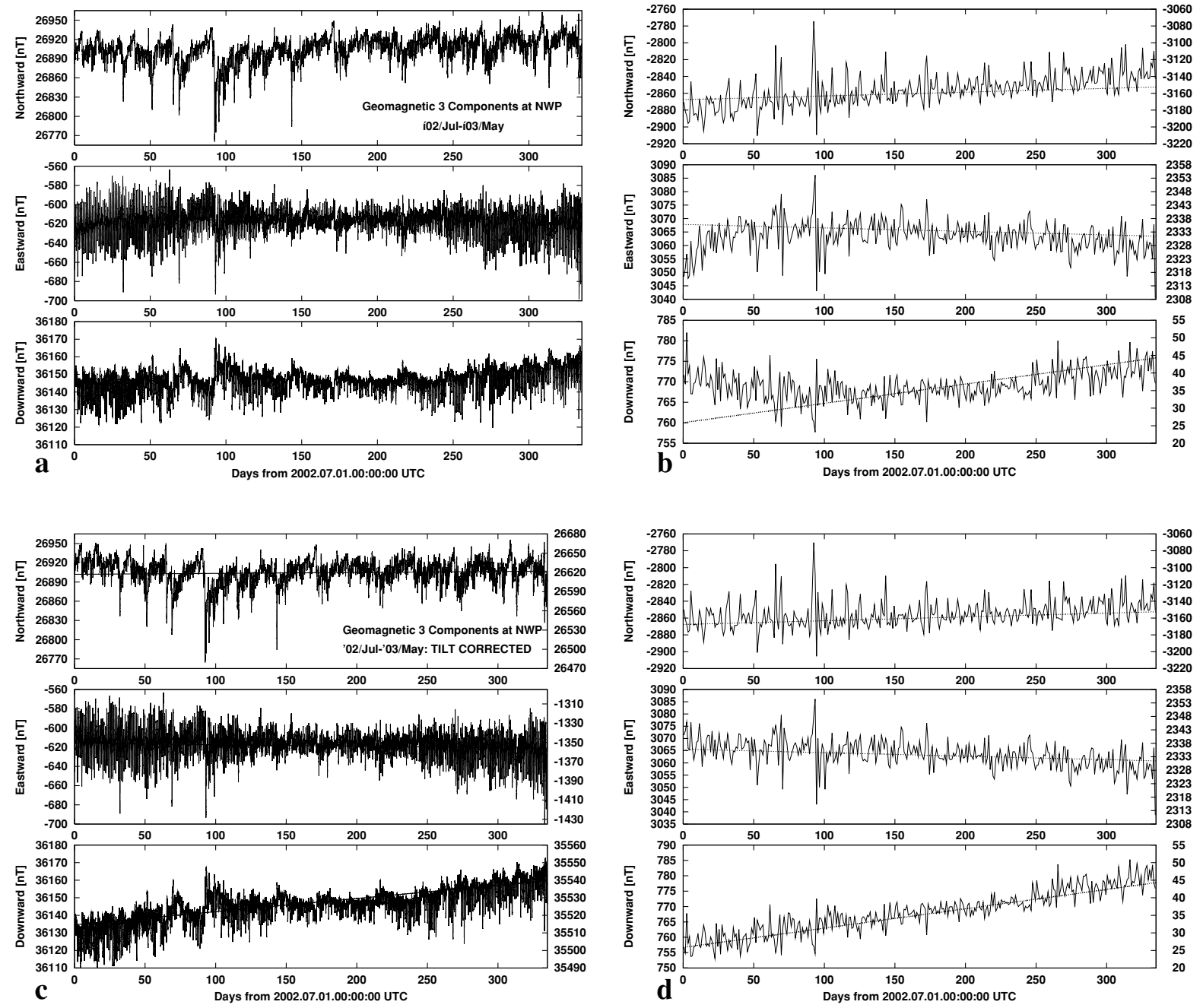

Fig. 9. (a) Variations of the geomagnetic three components at the seafloor without tilt correction. (b) Difference of the geomagnetic vector field between NWP and KAK. The dotted lines are the satellite predicted secular differences of the two sites. (c) The geomagnetic vector field at the seafloor when the tilt changes in Figs. 4(a) and 4(b) are considered. The thick lines are the satellite predicted secular variations at NWP. (d) Difference of the geomagnetic three components between NWP and KAK when the tilt correction is made.

SFEMS. The difference between the absolute geomagnetic total force and that synthesized from three geomagnetic components at the seafloor must not show a significant baseline change, if the three-component fluxgate magnetometer of SFEMS recorded the vector secular variation correctly. Figure 8 depicts how the two estimates of the geomagnetic total force behave at NWP. It is obvious that the difference is very stable for over half a year at the seafloor, and shows no signature of a systematic trend. This motivated us to compare the vector geomagnetic field observed at the seafloor with those estimated by the global models in the hope of resolving the vector secular variation.

The observed temporal variations of geomagnetic three components at NWP are shown for the 2002-2003 dataset in Fig. 9(a). Note that the variations are still raw in the sense that they are not corrected for the tilt changes in Figs. 4(a) and 4(b). If we take the same procedure to see the secular difference between NWP and KAK for each component as in the case of the geomagnetic total force, one ends up with Fig. 9(b) (upper right), which does not fit to the secular difference of what the magnetic satellite, Ørsted, predicts. However, if the seafloor vector field is corrected for the tilt changes in Figs. 4(a) and 4(b), it shows a very good fit to the predicted secular variation at NWP (Fig. 9(c)). The agreement is further confirmed by taking the vector secular difference with KAK again (Fig. 9(d)), which neatly fits to the observation.

The vector secular variation resolved in Fig. 9 seems promising. The stable baseline seen in Fig. 8 is a necessary condition to resolve the vector secular variation at the seafloor, but it is not sufficient. It is possible that the three measuring axes have baseline changes which cancel out with each other to give a seemingly nil instrumental drift in the synthetic total force. To ensure correct partitioning of the vector secular variation into each measuring axis, we should at least know the precise tilt change at the seafloor, the importance of which is clearly demonstrated by Fig. 9.

\section{Summary}

Here we reported details of the seafloor geomagnetic observation being conducted in the northwest Pacific, where needs to fill the gap in the existing geomagnetic observatory network have been keen for decades. The long-term geomagnetic observation at the seafloor was enabled by a newly 
developed geoelectromagnetic station, SFEMS. The twoyear long geomagnetic time series from SFEMS were examined using both global models and the presently operated geomagnetic observatory network on land. Our global time domain analysis confirmed the compatibility of the shortperiod temporal variations, with a minimum time scale of hours, observed at NWP with those predicted by the landbased geomagnetic network.

The linear time dependence of the geomagnetic main field was examined using predictions of the global models at NWP. It was shown that resolving the secular variation of not only the scalar field but also the vector field is now quite feasible even at the seafloor. However, it turned out essential to monitor the instrument's attitude at the seafloor as precisely as possible in order to yield the vector secular variation. Emphasis was also put on the importance of the secular variation seen in the downward geomagnetic component in terms of the 'core surface flow'. The vector secular variation thus revealed may give a constraint on delineating the core dynamics beneath the northwest Pacific, where contribution of non-dipole terms and their linear time derivatives seems curiously small.

Finally, we hope that the scalar and vector geomagnetic data at NWP thus verified will be considered in future global modeling of the geomagnetic field, since the data are shortly available via World Data Center for Geomagnetism at Kyoto University (http://swdcwww.kugi.kyoto-u.ac.jp/ mdplt/index.html) and via a site at Japan Agency for Marine-Earth Science and Technology (http://www.jamstec. go.jp/pacific21/emdmc/emdmc/).

Acknowledgments. Japan Agency for Marine-Earth Science and Technology is greatly acknowledged for their support at the time of the sea experiments. The skilful aid offered by R/V Kairei and ROV Kaiko was essential to complete this work. The authors are grateful to Heather McCreadie and two anonymous referees for their valuable comments indispensable to improve our original manuscript. This work was supported by Grant-in-Aid for Scientific Research, Ministry of Education, Culture, Sports, Science and Technology, Japan (Nos. 08NP1101 through 13NP1101).

\section{References}

Barker, F. S. and D. R. Barraclough, The effects of the non-uniform distribution of magnetic observatory data on secular variation models, Phys. Earth Planet. Inter., 37, 65-73, 1985.

Beranzoli, L., T. Braun, M. Calcara, P. Casale, A. De Santis, G. D’Anna, D. Di Mauro, G. Etiope, P. Favali, F. Frugoni, J.-L. Fuda, F. Gamberi, M. Marani, C. Millot, C. Montuori, and G. Smriglio, Mission results from the first GEOSTAR observatory (Adriatic Sea, 1998), Earth Planets Space, 55, 361-373, 2003.

Chave, A. D., D. J. Thompson, and M. E. Ander, On the robust estimation of power spectra, coherences, and transfer functions, J. Geophys. Res., 92, 633-648, 1987.

Copley, J., All wired up, Nature, 427, 10, 2004.
Hamano, Y., A new time-domain approach for the electromagnetic induction problem in a three-dimensional heterogeneous Earth, Geophys. J. Int., 150, 753-769, 2002.

International Association of Geomagnetism and Aeronomy (IAGA), Division V, Working Group VMOD: Geomagnetic Field Modeling, The 10th generation of International Geomagnetic Reference Field, Geophys. J. Int., 161, 561-565, 2005.

Jackson, A. and J. Bloxham, Mapping the fluid flow and shear near the core surface using the radial and horizontal components of the magnetic field, Geophys. J. Int., 132, 199-212, 1991.

Kerridge, D., INTERMAGNET: Worldwide near-real-time geomagnetic observatory data, Proc. Workshop on Space Weather, ESTEC, 2001.

Langel, R. A., R. H. Estes, G. D. Mead, E. B. Fabiano, and E. R. Lancaster, Initial geomagnetic field model from Magsat vector data, Geophys. Res. Lett., 7, 793-796, 1980.

Langel, R. A., R. T. Baldwin, and A. W. Green, Toward an improved distribution of magnetic observatories for modeling of the main geomagnetic field and its temporal change, J. Geomag. Geoelectr., 47, 475-508, 1995.

LeMouel, J. L., Outer core geostrophic flow and secular variation of Earth's magnetic field, Nature, 311, 734-735, 1984.

Macmillan, S., S. Maus, T. Bondar, A. Chambodut, V. Golovkov, R. Holme, B. Langlais, V. Lesur, F. Lowes, H. Lühr, W. Mai, M. Mandea, N. Olsen, M. Rother, T. Sabaka, A. Thomson, and I. Wardinski, The 9th generation international geomagnetic reference field, Phys. Earth Planet. Inter., 140, 253-254, 2003.

Mandea, M. and S. Macmillan, International Geomagnetic Reference Field - the eighth generation, Earth Planets Space, 52, 1119-1124, 2000.

Nakanishi, M. and E. L. Winterer, Tectonic history of the Pacific-FarallonPhoenix triple junction from Late Jurassic to Early Cretaceous: An abandoned Mesozoic spreading system in the Central Pacific Basin, $J$. Geohpys. Res., 103, 12453-12468, 1998.

Neubert, T., M. Mandea, G. Hulot, von R. Frese, F. Primdahl, J. L. Jørgensen, E. Friis-Christensen, P. Stauning, N. Olsen, and T. Risbo, Ørsted satellite captures high-precision geomagnetic field data, Eos, Trans. Amer. Geophys. Union, 82, 81-88, 2001.

Olsen, N., A model of the geomagnetic field and its secular variation for epoch 2000 estimated from Ørsted data, Geophys. J. Int., 149, 454-462, 2002.

Overhauser, A. W., Polarization of nuclei in metals, Phys. Rev., 92, 411415, 1953.

Reigber, C., H. Luhr, and P. Schwintzer, CHAMP mission status, Adv. Space Res., 30, 129-134, 2002.

Roberts, P. and S. Scott, On the analysis of the secular variation I. A hydrodynamic constraint: theory, J. Geomag. Geoelectr., 17, 137-151, 1965.

Toh, H. and Y. Hamano, The first realtime measurement of seafloor geomagnetic total force-Ocean Hemisphere Project Network, J. Japan Soc. Mar. Surv. Tech., 9, 1-23, 1997.

Toh, H., T. Goto, and Y. Hamano, A new seafloor electromagnetic station with an Overhauser magnetometer, a magnetotelluric variograph and an acoustic telemetry modem, Earth Planets Space, 50, 895-903, 1998.

Toh, H., Y. Hamano, M. Ichiki, and H. Utada, Geomagnetic observatory operates at the seafloor in the Northwest Pacific Ocean, Eos, Trans. Amer. Geophys. Union, 85, 467-473, 2004.

Voorhies, C. V. and G. E. Backus, Steady flows at the top of the core from geomagnetic field models: The steady motions theorem, Geophys. Astrophys. Fluid Dyn., 32, 163-173, 1985.

Whaler, K., Does the whole of the Earth's core convect?, Nature, 287, 528$530,1980$.

H. Toh (e-mail: toh@sci.u-toyama.ac.jp), Y. Hamano, and M. Ichiki 